\title{
A Conserved Region Common to Chikungunya (ChikV), Dengue (DenV) and Zika Viruses (ZikV): Potential as a Tool for Simultaneous Diagnosis and Therapeutics of the Three Viruses [Short Communication]
}

\author{
Chetan Datta Poduri \\ Independent Researcher, India
}

Received February 1, 2020; Revised April 14, 2020; Accepted April 27, 2020

Copyright $\odot 2020$ by authors, all rights reserved. Authors agree that this article remains permanently open access under the terms of the Creative Commons Attribution License 4.0 International License

\begin{abstract}
There is an increasing need to develop strategies for simultaneous detection of Chikungunya (ChikV), Dengue (DenV) and Zika Virus (ZikV) owing to their shared transmission ecology. Towards this aim, nine reference sequences of ChikV, DenV (1 - 4) and ZikV were aligned using ClustalW Omega Software available at the European Bioinformatics Institute (EBI) website. Internet search engines like Google (Scholar), PubMed, JSTOR, and ProQuest Central were used for literature search. Also, Conserved Domain Database (CDD) (NCBI) and ZikaVR (IMTECH, C'garh) formed constant references. One conserved region, 21 amino acids (aa) in length, common to the three viruses mapping to the Core protein of ChikV (aa 211 - 231); and the NS3 proteins of DenV (1 - 4) (aa 1608 - 1628) and ZikV (aa 1631/35 — $1651 / 55$ ) has been identified by the current author in this study. This region is the part of a protease in all the three viruses studied. Thus, this conserved region can form one diagnostic tool/probe for simultaneous detection of ChikV, DenV and ZikV. Additionally, as this region is conserved, it may form one therapeutic target.
\end{abstract}

Keywords ChikV, DenV, ZikV, Simultaneous, Diagnosis, Conserved, Core, NS3

\section{Introduction}

Recent observations of the World Health Organization (WHO) indicate that the disease dynamics of Zika Virus (ZikV) and Dengue Virus (DenV) seem to be working in tandem. Note that after the year 2016, when there was an epidemic with $\mathrm{ZikV}$, the number of DenV cases were relatively down [1, 2]. Also, ZikV, DenV and a third virus, the Chikungunya Virus (ChikV), share epidemiological parameters like transmission ecology [2, 3]. India is recognised as an endemic country to the viruses in context [4]. Furthermore, there are reports of the three viruses being isolated from a single mosquito and reports of natural co-infections with any two or all the three viruses in humans [5, 6, 7]. Additionally, the symptoms of many pathogenic infections overlap with those of the infections caused by either ChikV, DenV or ZikV. Thus, there is an urgent need for developing strategies for simultaneous diagnosis of the three viruses. Such a work would initially involve identification of regions that are common and conserved to the three viruses being studied for the present.

\section{Materials and Methods}

\subsection{Literature Search}

Literature search was performed using internet search engines like Google (Scholar), PubMed, JSTOR, and ProQuest Central (author's personal membership at the British Council Library, Hyderabad). In addition to this, Conserved Domain Database (CDD) at the National Center for Biotecnology Information (NCBI) and ZikaVR database maintained by IMTECH, Chandigarh, India, formed constant references.

\subsection{Reference Sequences}

Characteristics of ChikV, DenV and ZikV are summarized as table 1 . 
A Conserved Region Common to Chikungunya (ChikV), Dengue (DenV) and Zika Viruses (ZikV):

Potential as a Tool for Simultaneous Diagnosis and Therapeutics of the Three Viruses [Short Communication]

Table 1. Characteristics of the three viruses considered in the present study

\begin{tabular}{|c|c|c|c|}
\hline $\begin{array}{c}\text { Virus } \rightarrow \\
\text { Characteristic } \downarrow\end{array}$ & $\begin{array}{c}\text { Chikungunya Virus } \\
\text { (ChikV) }\end{array}$ & Dengue Virus (DenV) & Zika Virus (ZikV) \\
\hline Genus & Alphavirus & Flavivirus & Flavivirus \\
\hline Family & Togaviridae & Flaviviridae & Vertebrates \\
\hline Natural Host & Vertebrates & humans, monkeys \\
\hline $\begin{array}{c}\text { Vector / } \\
\text { Reservoir }\end{array}$ & Aedes sp. (Mosquito) & Aedes sp. (Mosquito) & Aosquito) \\
\hline Clinical Disease & $\begin{array}{c}\text { Unsymptomatic rash, } \\
\text { arthralgia, fever }\end{array}$ & $\begin{array}{c}\text { Dengue (with or without symptoms) \& Severe } \\
\text { Dengue - characteristic high fever, rash }\end{array}$ & $\begin{array}{c}\text { Zika fever (acute fever and rash) often } \\
\text { self-limiting and asymptomatic }\end{array}$ \\
\hline Nucleic Acid & $(+)$ ss RNA & $(+)$ ss RNA & $(+)$ ss RNA \\
\hline
\end{tabular}

Legend. RNA - Ribonucleic acid; ss - Single stranded; Other abbreviations carry their usual significance.

Table 2. Details of the reference sequences used in the present study

\begin{tabular}{|c|l|c|c|}
\hline Sl. $\mathbf{N}^{\mathbf{*}}$ & Virus & Protein & Accession Number \\
\hline 1 & Chikungunya Virus (ChikV) & Structural & ABN04200 \\
\hline 2 & Chikungunya Virus (ChikV) & Structural & ATW74975 \\
\hline 3 & Chikungunya Virus (ChikV) & Structural & NP_690589 \\
\hline 4 & Dengue Virus 1 (DenV 1) & Polyprotein & NP_659433 \\
\hline 5 & Dengue Virus 2 (DenV 2) & Polyprotein & NP_056776 \\
\hline 6 & Dengue Virus 3 (DenV 3) & Polyprotein & YP_001621843 aa \\
\hline 7 & Dengue Virus 4 (DenV 4) & Polyprotein & NP_073286 aa \\
\hline 8 & Zika Virus (ZikV) & Polyprotein & 3390 aa \\
\hline 9 & Zika Virus (ZikV) & Polyprotein & YP_002790881 \\
\hline
\end{tabular}

Legend. aa - amino acid; Other abbreviations carry their usual significance.

Towards the present aim, nine reference sequences of ChikV, DenV (1 - 4) and ZikV were considered and accessed from the NCBI-PubMed databases at https://www.ncbi.nlm.nih.gov/pubmed/. Details of the reference sequences used in the present study are given as Table 2.

\subsection{Alignment of the Sequences}

The nine reference sequences were aligned using ClustalW Omega Software available at the European Bioinformatics Institute (EBI) website (https://www.ebi.ac.uk/). Default parameters were used for aligning the sequences.

\subsection{Identification of Conserved Region Common to the Three Viruses Being Studied}

From the alignment output, a conserved region of 21 amino acids in length was identified manually without the help of any software. Figure 1 shows the conserved region in the aligned sequences. Figure 2 shows the identification of hypervariable regions (HVR) in the three viruses. 


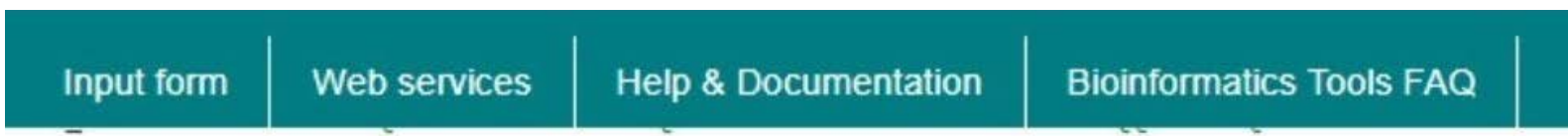

ABN94200.1

ATW74975.1

NP_690589.2

YP_ 002790881.1

AWH 65848.1

NP_073286.1

NP_ 056776.2

NP_059433.1

YP_001621843.1

$$
\therefore \quad: \quad * \quad: \quad \therefore: . .
$$

ABN 04200.1

ATW74975.1

NP_690589.2

YP_.02790881.1

AwH65848.1

NP_073286.1

NP_056776.2

NP_059433.1

YP_ø01621843.1

............. YACLVGDKVMKPAHVKGTIDNADLAK-- LAFKRSSKYDLEC-AQ............ YACLVGDKVYKPAHVKGTIDNADLAK-- LAFKRSSKYDLEC-AQ-

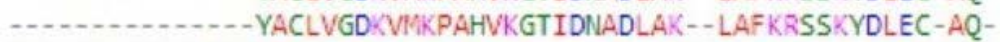
MQEGVFHTPWHVTKGAALRSGEGRLDPYUGDVK---QDLVSYCGPWKLDAAUIDGLSEVQL MQEGVFHTHWHVTKGSALRSGEGRLDPYIGDVK---QDLVSYCGPWKLDAAWDGHSEVQL HIIEGVFHTMWHVTRGSVICHETGRLEPSWADVR---NDMISYGGGWRLGDKWDKEEDVQV YKEGTFHTPWHVTRGAVLMHKGKRIEPSWADVK - - KDLISYGGGWKLEGEUKEGEEVQV FQEGVFHTPWHVTRGAVLMYQGKRLEPSWASVK---KDLISYGGGWRFQGSWNAGEEVQV QKEGVFHTIWIHVTRGAVLTHNGKRLEPNWASVK---KDLISYGGGWRLSAQUQKGEEVQV

ABNO4200.1

ATW74975.1

NP_690589.2

YP_002790881.1

AWH65848.1

NP_073286.1

NP_056776.2

NP_059433.1

YP_001621843.1

$$
\therefore * \quad * \text { * : : : .* }
$$

IPVHYKSDASKFTHEXPEGYNUHHGAVOYSGGRFTIPTGAGKP GDSGRPIFDNKGRVA IPVH MKSDASKFTHEKPEGYYNUHHGAVQYSGGRFTIPTGAGKP GDSGRPIFDNKGRVWA IPVHIKSDASKFTHEKPEGYNIVHHGAVQYSGGRFTIPTGAGKP GDSGRPIFDNKGRVA LAVPP-GERAR - ....... NIOTLPGIFKTKDGDIGAVALDYPA GTSGSPILDKCGRVIG LAVPP-GERAR-....--NIQTLPGIFKTKDGDIGAVALDYPAGTSGSPILDKCGRVIG LAIEP-GKNPK-......-HVQTKPGLFKTLTGEIGAVTLDFKP GTSGSPIINRKGKVIG LALEP-GKNPR….....AVQTKPGLFKTNAGTIGAVSLDFSP GTSGSPIIDKKGKWG IAVEP-GKNPK-.....-NVQTAPGTFKTPEGEVGAIALDFKP GTSGSPIVNREGKIVG IAVEP-GKNPK-...--NFQTTPGTFQTTTGEIGAIALDFKP GTSGSPIINREGKWG

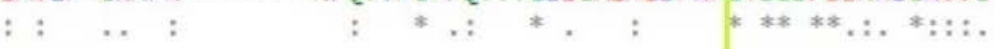

IVLGG ANEGARTALSWTWNKDIVTKITPEGAEEIS - - LAIPVMCLLANTTFPCSQPPCA IVLGG ANEGARTALSWTUNKDIVTKITPEGAEEUS - - LAIPVMCLLANTTFPCSOPPCI

IVLGG ANEGARTALSWTUNKDIVTKITPEGAEEWS- - LAIPVMCLLANTTFPCSQPPCI LYGNG WI KNGSWSAITQGKREEETPVECFEPSMLKKKQLTVLDLHPGAGK---TRRVL LYGNG NIKNGSYVSAITQGRREEETPVECFEPSMLKKKQLTVLDLHPGAGK---TRRVL LYGNG WTKSGDWSAITQAERIGE-PDYEVDEDIFRKKRLTIMDLHPGAGK---TKRIL LYGNG NTRSGAYVSAIAQTEKSIE-DNPEI EDDIFRKRRLTIMDLHPGAGK-.-TKRYL LYGNG NTTSGTYSAIAQAKASQEGPLPEI EDEVFRKRNLTIMDLHPGSGK - - TRRYL LYGNG NTKNGGYSSIAQTNAEPDGPTPELEEEMFKKRNLTIMDLHPGSGK---TRKYL

Figure 1. Conserved region common to ChikV, DenV and ZikV identified in the present study (box) 
identified region shown in figure 1 and table 3 is conserved among all the three viruses in context, which this publication does.

A hypervariable region (HVR) is characteristic of RNA viruses. It may be noted that the three viruses are RNA viruses. In the present study it was also observed that all the three viruses exhibit variability in their sequences (Figure 2; data of individual virus strains comparisons not shown). Given these observations, the importance of identifying a conserved region in ChikV, DenV and $\mathrm{ZikV}$ gains momentum.

\section{Conclusions}

To the best of the authors' knowledge, this is the first publication identifying a conserved region that is common to ChikV, DenV and ZikV. This conserved region can form one diagnostic tool/probe for simultaneous detection of ChikV, DenV and ZikV. Additionally, as this region contains one potential phosphorylation site, it may form one therapeutic target against ChikV, DenV and ZikV.

\section{Acknowledgements}

This is a self-financed study.

The author wishes to acknowledge the anonymous reviewers for the constructive suggestions.

\section{REFERENCES}

[1] Anonymous. Dengue and Severe Dengue. World Health Organization. https://www.who.in 15 April 2019. Accessed online 22 June 2019 and again on 01 Dec 2019.

[2] Ramos-Castañeda J,Flavia Barreto dos Santos,Ruth Martínez-Vega,Josélio Maria Galvão de Araujo,Graham Joint,Elsa Sarti. Dengue in Latin America: Systematic Review of Molecular Epidemiological Trends. PLoS Neglected Tropical Diseases, Jan 2017; 11 (1): e0005224, 2017.

[3] Kamaraj US, Tan JH, Xin Mei O, Pan L, Chawla T, Uehara A, et al. (2019) Application of a targeted-enrichment methodology for full-genome sequencing of Dengue 1-4, Chikungunya and Zika viruses directly from patient samples. PLoS Neglected Tropical Diseases, Apr 2019; 13(4): e0007184.https://doi.org/10.1371/journal.pntd.0007 184, 2019.

[4] Poduri CD. Exotic human viruses of India. Virus Disease, 2019. https://doi.org/10.1007/s13337-019-00523-8. Web/P ublished Abstract, 2019.

[5] Bagno FF, Figueiredo MM, Villarreal J, Pereira GC, Godoi LC, da Fonseca FG. Undetected Chikungunya virus co-infections in a Brazilian region presenting hyper-endemic circulation of Dengue and Zika. Journal of
Clinical Virology. 2019 Apr; 113: 27-30. doi: 10.1016/j.jc v.2019.02.006, 2019

[6] Magalhaes T, Braga C, Cordeiro MT, Oliveira ALS, Castanha PMS, Maciel APR, et al. (2017) Zika virus displacement by a chikungunya outbreak in Recife, Brazil. PLoS Neglected Tropical Diseases 11(11): e0006055. https://doi.org/10.1371/journal.pntd.0006055, 2017

[7] Rückert C, Weger-Lucarelli J, Garcia-Luna SM, et al. Impact of simultaneous exposure to arboviruses on infection and transmission by Aedes aegypti mosquitoes. Nature Communications, 2017 May 19; 8:15412. doi: 10.1 038/ncomms15412, 2017

[8] Goh LYH,Hobson-Peters J,Prow NA et al. The Chikungunya Virus Capsid Protein Contains Linear B Cell Epitopes in the $\mathrm{N}$ - and C-Terminal Regions that are Dependent on an Intact C-Terminus for Antibody Recognition. Viruses. 2015; 7 (6): 2943-2964. Published 2015 Jun 8. doi: 10.3390/v7062754, 2015

[9] Keating JA and Striker R. Phosphorylation events during viral infections provide potential therapeutic targets. Reviews in Medical Virology, 2012 May; 22(3), pp 166-181, 2012

[10] Gupta AK, Kaur K, Rajput A, et al. ZikaVR: An Integrated Zika Virus Resource for Genomics, Proteomics, Phylogenetic and Therapeutic Analysis. Scientific Reports | 6:32713 | DOI: 10.1038/srep32713, 2016

[11] Lura T, Su T, and Brown MQ. Preliminary evaluation of Thermo Fisher TaqMan ${ }^{\circledR}$ Triplex q-PCR kit for simultaneous detection of chikungunya, dengue, and Zika viruses in mosquitoes. Journal of Vector Ecology; 44 (1), pp 205 - 209, 2019 